\title{
Relación entre la ansiedad rasgo y los estilos de afrontamiento al estrés en conductores infractores de Lima Metropolitana
}

\author{
Relationship between trait anxiety and stress coping styles in offending \\ drivers in Metropolitan Lima \\ Marco Briozo A. ${ }^{1}$ \\ Universidad Nacional Mayor de San Marcos, Lima, Perú \\ (RECIBIDO 10/02/2015, AcEPTADo 15/06/2015)
}

\begin{abstract}
RESUMEN
La presente investigación es de tipo cuantitativo, descriptivo-correlacional, cuyo objetivo principal es encontrar la relación que existe entre la ansiedad rasgo y los estilos de afrontamiento al estrés en conductores infractores de Lima Metropolitana. Para dicho propósito se recurrirá al uso del test de ansiedad rasgo de Idare y al test de estilos de afrontamiento al estrés de COPE. Bajo un muestreo intencional y un estudio no experimental se pretende llegar a encontrar una correlación significativa y los niveles de estilos de afrontamiento más predominantes. Los resultados hallados demuestran que existe una correlación directa entre la ansiedad-rasgo y los estilos planificación de actividades, análisis de las emociones, negación y conductas inadecuadas. Una correlación inversa entre la ansiedad-rasgo y los estilos búsqueda de soporte social, búsqueda de soporte emocional, supresión de actividades competitivas, reinterpretación positiva de la experiencia, aceptación y distracción. No hay correlación entre las estrategias de afrontamiento al estrés de afrontamiento directo, retracción del afrontamiento y retorno a la religión.
\end{abstract}

Palabras clave: Estilo de afrontamiento, estrés, ansiedad.

\begin{abstract}
This research is quantitative, descriptive and correlational type whose principal aim is to find the correlation between trait anxiety and coping styles to stress in offending drivers in Metropolitan Lima.

For this purpose, the State-Trait Anxiety Inventory (STAI) and Stress Coping Styles Test by Cope will be used. Under an intentional sampling and a non-experimental study, it aims to find a significant correlation and the most predominant levels of coping styles. The research findings show a direct correlation between Trait Anxiety and the styles of Planning Activities, Analysis of Emotions, Negation and Inappropriate Behavior. On the other hand, it shows inverse correlation between Trait Anxiety and the styles of Search of Social Support,
\end{abstract}

1 E-mail: marcobriozo@gmail.com 
Search of Emotional Support, Competitive Activities Suppression, Positive Reinterpretation of Experience, Acceptance and Distraction. Finally, there is no correlation between stress coping strategies and Direct Coping, Coping Retraction and Return to Religion.

Keywords: Coping style, stress, anxiety.

\section{INTRODUCCIÓN}

La Organización Mundial de la Salud estimó que en el 2002 murieron 1.18 millones de personas por causa de choques en la vía pública, lo que significa una media de 3,242 fallecimientos diarios (OMS, 2004). A nivel de Perú, un estudio indicó que los accidentes de tránsito han provocado 17,025 muertos y 235,591 lesionados durante el periodo 2005 - 2009. Al analizar los accidentes de tránsito, uno se percata de la multifactorialidad de las variables que convergen, tal como lo señala Haddon (1962; citado en OMS, 2004) bajo el enfoque sistémico: factores del entorno, condiciones ambientales de la carretera y normas sociales; factores del vehículo y equipo, el estado de auto y/o bus; y el factor humano, por parte del conductor.

De acuerdo a Ponce y col. (2006), los choferes del servicio público y particulares presentan una conducta desajustada, de alto riesgo y peligrosa, dentro de los cuales se podría considerar la presencia de niveles elevados de ansiedad en los conductores. Siguiendo a Spielberger, concibe a la ansiedad como un estado transitorio y como un rasgo de personalidad, para él los estados de ansiedad se caracterizan por ser sentimientos de tensión y aprensión subjetivos conscientemente percibidos; en contraste, "la ansiedad como rasgo de la personalidad parecería implicar un motivo o disposición conductual adquirida que predispone al individuo a percibir un amplio rango de circunstancias objetivamente no peligrosas como amenazantes, y responder a ellas con reacciones de ansiedad - estado desproporcionadas en intensidad a la magnitud del peligro objetivo". Al investigar los factores que hacen que algunas personas se sientan agotadas y consumidas por los estresores y que otras personas se sientan estimuladas y excitadas por ellos, la capacidad de manejar el estrés depende del estilo personal y la personalidad; en otros términos, de qué manera el sujeto tiende a percibir e interpretar los acontecimientos productores de estrés y cómo responde ante ellos según los estilos de afrontamiento que posee. Carver y col. (1994) mencionan que el afrontamiento al estrés es la respuesta a una situación estresante y depende de cada uno de las experiencias anteriores, la autoapreciación de las propias capacidades y las motivaciones que influyen en este proceso activo de adaptación. Los estilos de afrontamiento al estrés hacen referencia a las distintas estrategias o alternativas de respuesta que tendrá la persona para afrontar diferentes situaciones estresantes. Según Carver, las estrategias son trece: afrontamiento directo (activo), planificación de actividades, supresión de actividades competitivas, retracción (demora) del afrontamiento, búsqueda de soporte social, búsqueda de soporte emocional, 
reinterpretación positiva de la experiencia, aceptación, retorno a la religión, análisis de las emociones, negación, conductas inadecuadas y distracción (afrontamiento indirecto). En relación con lo mencionado, es de interés para el presente estudio conocer cuál es la relación entre el nivel de ansiedad rasgo y los estilos de afrontamientos en los conductores infractores de Lima Metropolitana. Para ello se plantearon las siguientes hipótesis de investigación:

Hipótesis general: Existe relación entre la presencia de ansiedad rasgo y los estilos de afrontamiento al estrés en conductores infractores de Lima Metropolitana.

Hipótesis específicas: Hay un alto nivel de ansiedad rasgo en los conductores infractores de Lima Metropolitana. Hay bajos estilos de afrontamiento al estrés en los conductores infractores de Lima Metropolitana.

\section{MÉTODO}

\section{Alcance}

El tipo de estrategia que se siguió para alcanzar los objetivos propuestos corresponde a una investigación no experimental, ya que en este estudio no se pretende manipular o variar intencionalmente ninguno de los componentes de una variable independiente (Hernández, Fernández y Baptista, 2006).

\section{Diseño}

El diseño utilizado fue el no experimental, transeccional y correlacional, dado que no se manipuló variable alguna y la recolección de la información con respecto a las variables de estudio se efectuó en un periodo de tiempo determinado.

\section{Variables}

Variable de estudio 1: Ansiedad rasgo. Variable de estudio 2: Estilos de afrontamiento al estrés.

\section{Sujetos}

La muestra estuvo constituida por 120 conductores del transporte público con brevete AII B y AIII A que han realizado alguna infracción de tránsito.

\section{Técnica}

Muestreo no probabilístico intencional, con criterios de inclusión- exclusión.

\section{Procedimiento}

Se seleccionaron los test más apropiados para el tipo de muestra con la cual se trabajaría. Se seleccionó la institución o academia de manejo con mayor accesibilidad para la aplicación de los test. Se coordinó con las autoridades de las instituciones para la toma de los test. Se efectuó la capacitación del equipo de campo encargado de la administración de los instrumentos de evaluación. Se efectuó el procesamiento y análisis de la información recolectada. Se redactó el informe de investigación. 


\section{Instrumentos de evaluación}

\section{Instrumento 1}

\begin{tabular}{lll}
\hline Nombre & $:$ Inventario de ansiedad-estado y ansiedad-rasgo (Idare) \\
\hline Autores & $:$ Spielberger, Gorsuch \& Lushene \\
Año & $:$ 1970 \\
Objetivo & $:$ Evaluar la ansiedad estado y la ansiedad rasgo \\
Administración & $:$ Individual y grupal \\
Edad & $:$ A partir de los 12 años de edad en adelante \\
Duración & $:$ 15 minutos \\
Dimensiones & $:$ Ansiedad - estado y ansiedad - rasgo \\
\hline
\end{tabular}

\section{Instrumento 2}

\begin{tabular}{lll}
\hline Nombre & $:$ Escala de modos de afrontamiento al estrés (COPE) \\
\hline Autores & $:$ Carver, Scheiry Weintraub \\
Año & $:$ & 1989 \\
Objetivo & $:$ & Estimar trece modos de afrontamiento al estrés y dos funciones de afrontamiento \\
Administración & $:$ & Individual y colectiva \\
Edad & $:$ & 16 años a más \\
Duración & $:$ & 20 minutos aproximadamente. \\
& $\begin{array}{l}\text { Afrontamiento directo (activo), planificación de actividades, supresión de } \\
\text { actividades competitivas, retracción (demora) del afrontamiento, búsqueda de } \\
\text { soporte social, búsqueda de soporte emocional, reinterpretación positiva de la } \\
\text { experiencia, aceptación, retorno a la religión, análisis de las emociones, negación, } \\
\text { conductas inadecuadas, distracción (afrontamiento indirecto). }\end{array}$ \\
&
\end{tabular}

\section{RESULTADOS}

Los resultados se presentan mediante tablas, que corresponde principalmente a la parte inferencial.

\section{Estadística inferencial}

Para responder a la pregunta de investigación, se analizan los resultados por medio del paquete estadístico SPSS. No obstante, en primer lugar se tiene que analizar si los datos se procesan con estadísticos paramétricos o con estadísticos no paramétricos. Para ello se recurre al análisis del estadístico no paramétrico Kolmogorov-Smirnov, que indicará si los datos se distribuyen normalmente o no.

La siguiente tabla muestra los resultados: 
Tabla 1. Prueba de Kolmogorov-Smirnov para una muestra

Prueba de Kolmogorov-Smirnov para una muestra

\begin{tabular}{|c|c|c|c|c|c|c|c|c|c|c|c|c|c|c|}
\hline \multicolumn{15}{|c|}{ Prueba de Kolmogorov-Smirnov para una muestra } \\
\hline & 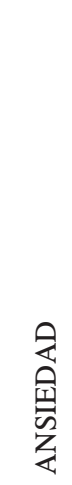 & 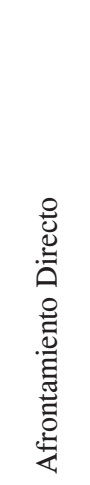 & 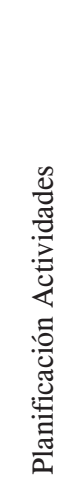 & 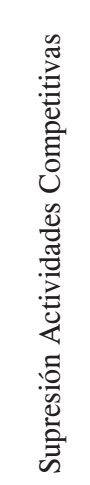 & 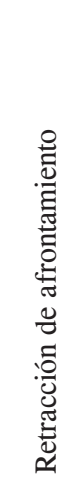 & 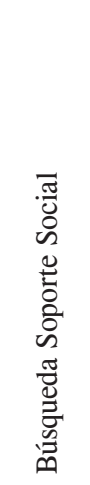 & 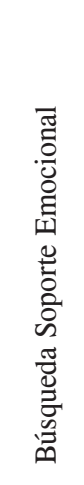 & 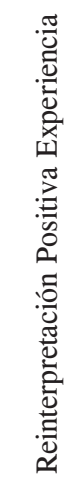 & 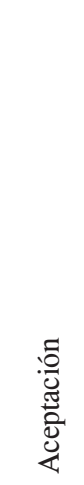 & 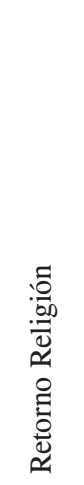 & 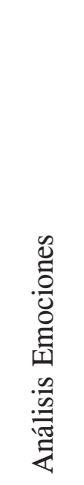 & 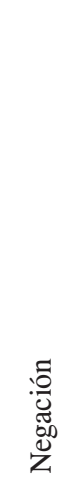 & 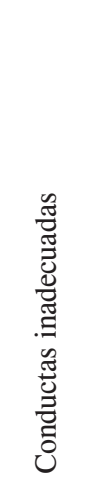 & 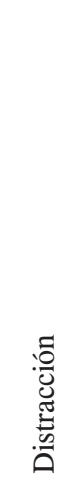 \\
\hline $\begin{array}{l}\mathrm{Z} \text { de } \\
\text { Kolmogorov- } \\
\text { Smirnov }\end{array}$ & 3.181 & 5.806 & 4.85 & 3.728 & 3.728 & 5.806 & 3.603 & 3.603 & 3.728 & 5.806 & 4.234 & 4.850 & 4.728 & 4.293 \\
\hline $\begin{array}{l}\text { Sig. } \\
\text { (bilateral) }\end{array}$ & .000 & .000 & .000 & .000 & .000 & .000 & .000 & .000 & .000 & .000 & .000 & .000 & .000 & .000 \\
\hline \multicolumn{15}{|c|}{ a. La distribución de contraste es la normal. } \\
\hline \multicolumn{15}{|c|}{ b. Se han calculado a partir de los datos. } \\
\hline
\end{tabular}

De lo anterior, se concluye que los datos (estrategias de afrontamiento y ansiedad) tienen una distribución no normal, por lo que se usarán estadísticos no paramétricos para analizar la correlación

En este caso, la pregunta general de investigación que pretende responder esta investigación fue: ¿Qué relación existe entre la ansiedad rasgo y los estilos de afrontamiento al estrés en conductores infractores de Lima Metropolitana? Para responderla se procedió a seleccionar el estadístico de correlación no paramétrico como es la Rho de Spearman. La siguiente tabla muestra los resultados de las correlaciones de la variable ansiedad y los trece estilos de afrontamiento:

Tabla 2. Correlaciones de estilos de afrontamiento y ansiedad rasgo

\begin{tabular}{lcc}
\hline \multicolumn{1}{c}{ Estrategias de afrontamiento } & Significación & Ansiedad rasgo \\
\hline \multirow{2}{*}{ Afrontamiento directo } & Coeficiente de correlación & 0 \\
\hline \multirow{2}{*}{ Planificación de actividades } & Sig. (bilateral) & .000 \\
\hline \multirow{2}{*}{ Supresión de actividades competitivas } & Coeficiente de correlación & $.913^{* *}$ \\
\cline { 2 - 3 } & Sig. (bilateral) & .000 \\
\cline { 2 - 3 } & Coeficiente de correlación & $-.398^{* *}$ \\
\hline
\end{tabular}




\begin{tabular}{|c|c|c|}
\hline \multirow{2}{*}{ Retracción del afrontamiento } & Coeficiente de correlación & 0 \\
\hline & Sig. (bilateral) & .000 \\
\hline \multirow{2}{*}{ Búsqueda de soporte social } & Coeficiente de correlación & $-.598^{* *}$ \\
\hline & Sig. (bilateral) & .000 \\
\hline \multirow{2}{*}{ Búsqueda de soporte emocional } & Coeficiente de correlación & $-.594^{* *}$ \\
\hline & Sig. (bilateral) & .000 \\
\hline \multirow{2}{*}{ Reinterpretación positiva de la experiencia } & Coeficiente de correlación & $-.594^{* *}$ \\
\hline & Sig. (bilateral) & .000 \\
\hline \multirow{2}{*}{ Aceptación } & Coeficiente de correlación & $-.398^{* *}$ \\
\hline & Sig. (bilateral) & .000 \\
\hline \multirow{2}{*}{ Retorno a la religión } & Coeficiente de correlación & -.133 \\
\hline & Sig. (bilateral) & .148 \\
\hline \multirow{2}{*}{ Análisis de emociones } & Coeficiente de correlación & $.679^{* *}$ \\
\hline & Sig. (bilateral) & .000 \\
\hline \multirow{2}{*}{ Negación } & Coeficiente de correlación & $.522^{* *}$ \\
\hline & Sig. (bilateral) & .000 \\
\hline \multirow{2}{*}{ Conductas inadecuadas } & Coeficiente de correlación & $.351^{* *}$ \\
\hline & Sig. (bilateral) & .000 \\
\hline \multirow{2}{*}{ Distracción } & Coeficiente de correlación & $-.773^{* *}$ \\
\hline & Sig. (bilateral) & .000 \\
\hline
\end{tabular}

De los resultados anteriores se encuentra que la información es significativa a un nivel de 0.05 . Por tanto, se encontró una correlación directa entre la ansiedadrasgo y los estilos planificación de actividades, análisis de las emociones, negación y conductas inadecuadas. Esto quiere decir que a mayor ansiedad, existen mayores niveles de planificación de actividades, análisis de emociones, conductas inadecuadas y también negación de la problemática.

Por otro lado, se encontró una correlación inversa entre la ansiedad-rasgo y los estilos búsqueda de soporte social, búsqueda de soporte emocional, supresión de actividades competitivas, reinterpretación positiva de la experiencia, aceptación y distracción. Esto quiere decir que a mayores niveles de ansiedad, existen menores niveles de soporte social, soporte emocional, supresión de actividades competitivas, reinterpretación positiva de la experiencia, aceptación y distracción.

Finalmente, no se encontró correlación entre las estrategias de afrontamiento al estrés de afrontamiento directo, retracción del afrontamiento y retorno a la religión.

Por otro lado, para dar respuesta a las preguntas específicas de investigación, se procedió a obtener los descriptivos para cada una de las variables. Las preguntas fueron:

¿Cuáles son los niveles de ansiedad rasgo en los conductores infractores de Lima Metropolitana? 
Tabla 3. Ansiedad rasgo

\begin{tabular}{cccccc}
\hline \multicolumn{7}{c}{ ANSIEDAD } \\
\hline & Frecuencia & Porcentaje & Porcentaje válido & Porcentaje acumulado \\
\hline Válidos & bajo & 36 & 29.0 & 29.0 & 29.0 \\
\hline & medio & 76 & 61.3 & 61.3 & 90.3 \\
\hline & alto & 12 & 9.7 & 9.7 & 100,0 \\
\hline & Total & 124 & 100.0 & 100.0 & \\
\hline
\end{tabular}

En el siguiente gráfico se aprecia que existen mayores porcentajes para las personas con un nivel de ansiedad medio, mientras que menos casos con un nivel de ansiedad alto.

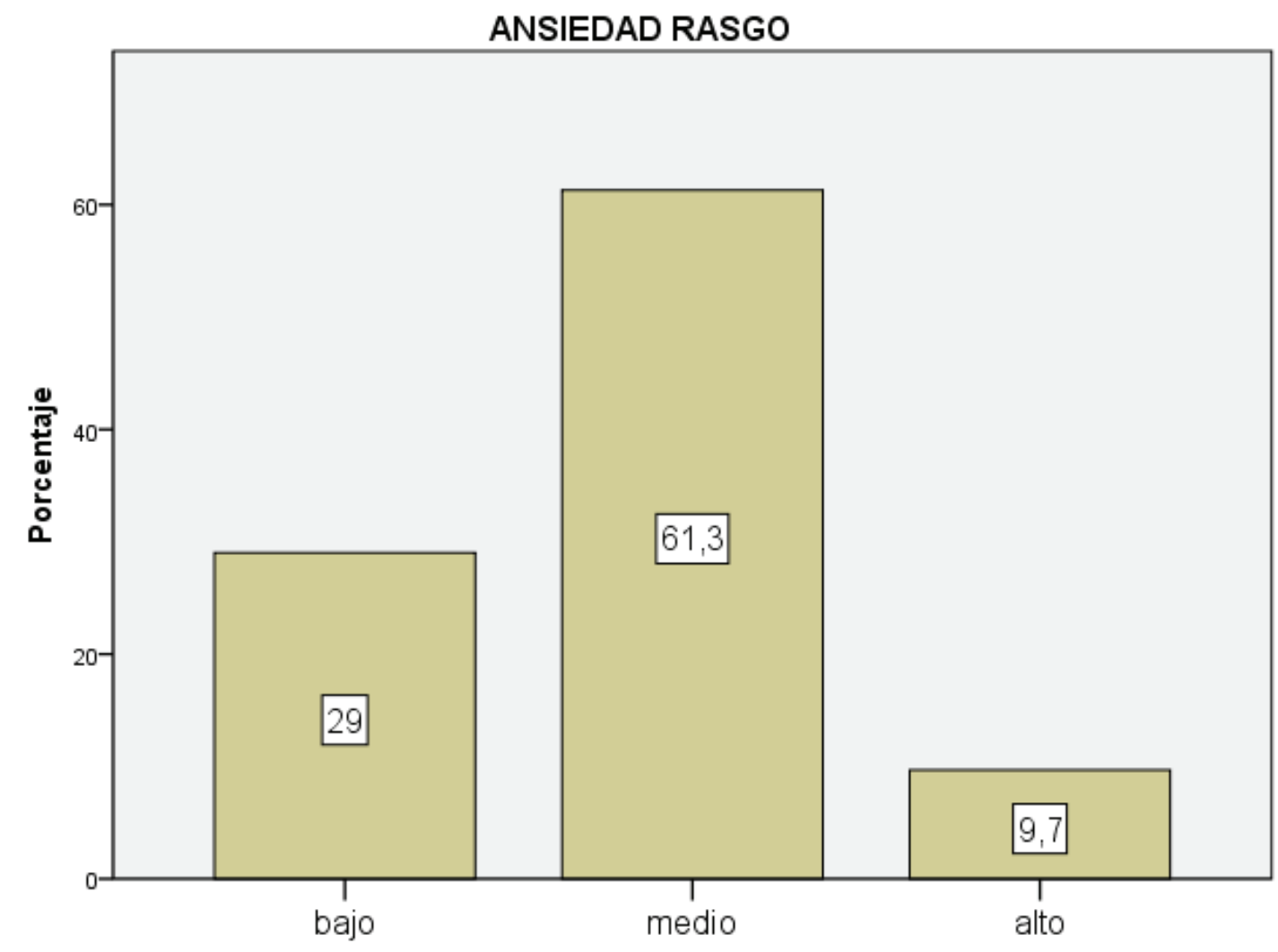

Figura 1. Ansiedad rasgo

¿Cuáles son los estilos de afrontamiento al estrés en los conductores infractores de Lima Metropolitana? 
Los más usados por los conductores son:

Tabla 4. Estilos de afrontamiento al estrés

\begin{tabular}{clcc}
\hline \multicolumn{4}{c}{ ESTILOS DE AFRONTAMIENTO AL ESTRÉS } \\
\hline & Frecuencia & Porcentaje \\
\hline Válidos & Afrontamiento activo & 124 & $100 \%$ \\
\hline & Planificación & 43 & $35 \%$ \\
\hline Postergación del afrontamiento & 31 & $25 \%$ \\
\hline Acudir a la religión & 62 & $50 \%$ \\
\hline Razones instrumentales & 59 & $47.5 \%$ \\
\hline Razones emocionales & 28 & $22.5 \%$ \\
\hline Aceptación & 28 & $22.5 \%$ \\
\hline Evadir el afrontamiento & 31 & $25 \%$ \\
\hline Enfocar y liberar emociones & 56 & $45 \%$ \\
\hline Desentendimiento mental & 47 & $37.5 \%$ \\
\hline Supresión de actividades competentes & 43 & $35 \%$ \\
\hline Negación & 47 & $37.5 \%$ \\
\hline Desentendimiento conductual & 43 & $35 \%$ \\
\hline
\end{tabular}

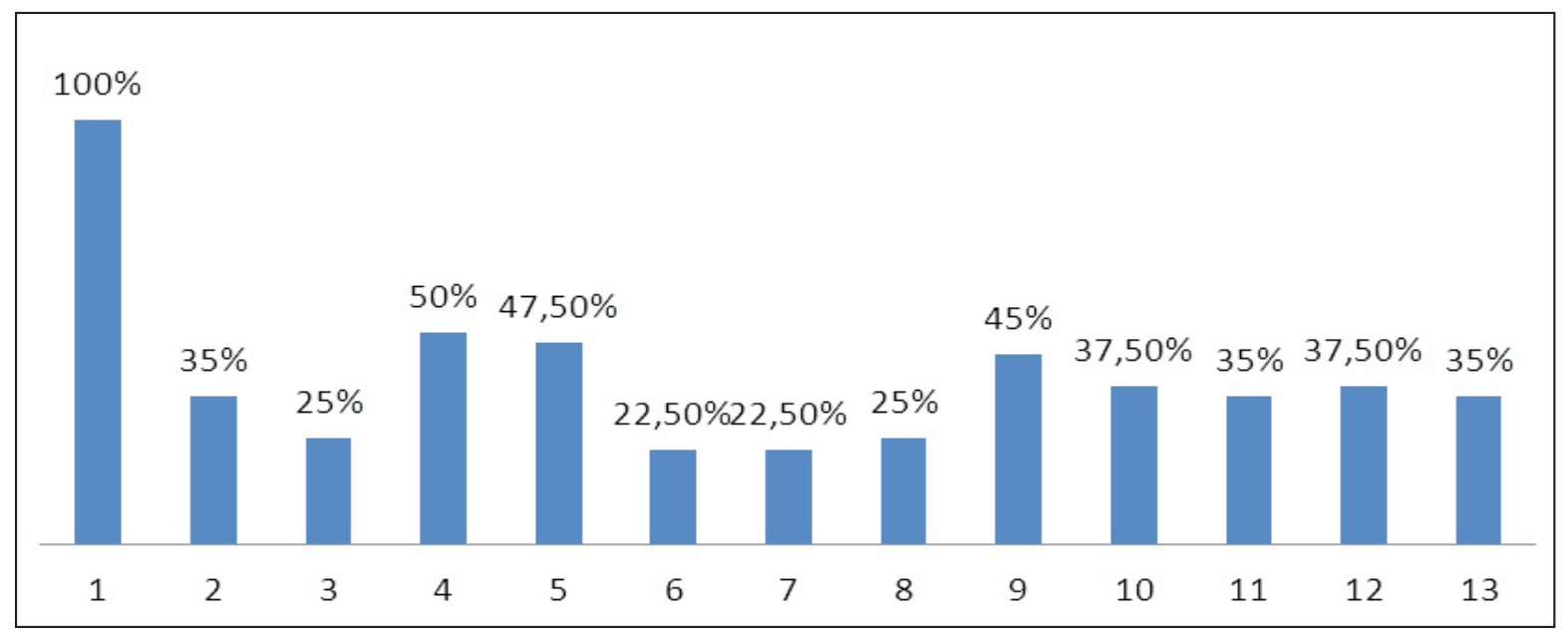

Figura 2. Estilos de afrontamiento

El estilo de afrontamiento "activo", seguido del estilo de afrontamiento de "acudir a la religión" y, ligeramente debajo, "búsqueda de apoyo social (razones instrumentales)". Los estilos menos usados fueron: "búsqueda de apoyo social (razones emocionales)" y "aceptación".

Finalmente se concluye que se encontraron estadísticos significativos, donde se encontró correlación entre las principales variables del estudio. 


\section{DISCUSIÓN}

La actividad de manejar es un proceso motor que involucra no solamente actividades corporales, muchas veces automáticas y repetitivas, sino que se pone en juego una serie de procesos cognitivos y emocionales.

Se encontró una correlación directa entre la ansiedad-rasgo y los estilos planificación de actividades, análisis de las emociones, negación y conductas inadecuadas. Cuando los conductores se ven expuestos a ansiedad en situaciones de tráfico, experimentan mayor necesidad de solucionar el problema lo más pronto posible, ya que tienen que cumplir con sus actividades dentro de un tiempo esperado. Por ello se ven en la obligación de planear rápidamente alternativas de solución, que los lleva a experimentar diferentes emociones variadas. En plena frustración buscan solucionar lo más rápido posible el suceso y a veces niegan parte de su culpa o responsabilidad quizá realizando algunas conductas inadecuadas.

Por otro lado, se encontró una correlación inversa entre la ansiedad-rasgo y los estilos búsqueda de soporte social, búsqueda de soporte emocional, supresión de actividades competitivas, reinterpretación positiva de la experiencia, aceptación y distracción. En los casos de accidentes, los conductores no buscan caerle bien a la otra persona, por eso decrece la necesidad de soporte social o emocional, pues no está puesto en juego sus emociones ni sentimientos; tampoco hay la necesidad de mostrar competencia en quién manejó mejor o no, así como la de procesarlo de un modo positivo, a manera de aprendizaje, ya que es un suceso real lo que se está observando. Los estilos de aceptación y distracción también disminuyen mientras la ansiedad aumenta, ya que no se busca la aceptación de la otra persona, por el contrario, se le hace saber lo mal que manejó para que haya existido ese problema y se le aborda directamente, no a modo de distracción, para que la persona esté bien concentrada en lo que se le dice.

Finalmente no se encontró correlación entre las estrategias de afrontamiento al estrés de afrontamiento directo, retracción del afrontamiento y retorno a la religión. Se asume que esto sucedió, ya que fueron estrategias que estuvieron presentes en su mayoría como otras que no estuvieron presentes para nada influyeron en la variable de ansiedad. Por ejemplo, el estilo de afrontamiento directo fue un estilo usado por todos, pero el estilo de retorno a la religión no fue aplicado por algún conductor. Es decir, su uso o aplicación fue nulo.

También sería bueno preguntarse qué es lo que causa ansiedad en los conductores. Muchas pueden ser las especulaciones, pero lo cierto es que en una investigación realizada por Lima y Juárez (2008), quienes buscaron identificar los estresores percibidos por los conductores de transporte público, se encontraron quince estresores principales en la muestra de estudio, entre los que se destaca como principales: el tráfico, la presión de tiempo que tienen los conductores y la presión por completar una suma de dinero determinada diariamente, los cuales concordaban con otros estudios similares. Ello genera que el conductor muchas 
veces sea percibido como una persona con un maltrato y que no guarde empatía con las personas que se encuentra trasladando en su vehículo. Por ello, dando importancia a este aspecto, fue que Ignacio y Catalina (2010) se interesaron en medir las características de hostilidad, ira, extraversión e indicadores de malas prácticas de conducción (accidentes y multas), y encontraron altos niveles de estas características en los conductores de vehículos públicos más que privados.

Ponce y col. (2006) desarrollaron un estudio con el propósito de conocer los estilos de comportamiento en contextos de tráfico que presentan los automovilistas de servicio público y los choferes particulares de Lima Metropolitana, donde encontraron que los choferes interprovinciales presentan comparativamente estilos de comportamiento positivos en contextos del tráfico que le permiten conducir bajo una percepción y aceptación del riesgo adecuada; son precavidos, atentos y responsables, con alto nivel de seguridad. En cambio, en la muestra de choferes de servicio público y particular predomina el estilo de conducta desajustada, de alto riesgo y peligrosa, no en su totalidad pero aparentemente con menos responsabilidad que el grupo anteriormente mencionado. Tampoco se quiere decir que se cae en el otro extremo. Ello demuestra que los conductores del ámbito urbano presentan poca tolerancia y estilos de afrontamiento en comparación con los choferes interprovinciales, de allí que los accidentes sean más numerosos en Lima Metropolitana y esté perjudicando y atentando contra la vida de diversos peatones, justamente porque estos conductores presentan poca sensibilización frente a la responsabilidad de conducir.

Choquehuanca, Cárdenas, Collazos y Mendoza (2010) encontraron que el $63.8 \%$ de los accidentes sucedía en Lima; las principales causas fueron el exceso de velocidad y la imprudencia del conductor, considerando al choque el tipo de accidente más común (57\%), justamente lo que se venía sustentando anteriormente. De modo complementario, Ignacio y Catalina (2010) encontraron que un mayor puntaje en la dificultad de conducir iba relacionado con mayor hostilidad, ira, accidentes y multas, lo que también respalda y se convierte en una evidencia más para esta investigación.

Otro componente importante a tener en consideración es la cantidad de horas que se encuentran frente al volante. Según los datos descriptivos que se analizaron, se encontró que existen más casos de participantes con 08 a 09 horas de manejo, seguido de los que manejan 10 horas y de los que manejan 07 horas. En menor medida, se encontraron los participantes que manejan de 04 a 06 horas, siendo justamente este último tramo de horas la más recomendable. La actividad de conducir para muchos representa una actividad estresante pues hay que poner en juego todos los sentidos y requiere una atención sostenida y altos niveles de concentración, que a su vez involucra dejar de hacer otras actividades paralelas como leer periódico o contestar el teléfono, dejar de comer, entre otras conductas inadecuadas muy frecuentes en los conductores de Lima Metropolitana. Y es que la cultura del manejo está poco promovida en el entorno limeño y es un tema que causa poca sensibilización y preocupación a los conductores de la urbe. 
Por otro lado, se rechaza la hipótesis específica de investigación que planteaba altos niveles de ansiedad. Según los resultados encontrados, vale resaltar que los niveles de ansiedad en los conductores son medios, sin presentar muchos niveles de ansiedad altos. Ello debido a estar tanto tiempo en la misma actividad, pues poseen una adecuación al estrés que hace que sientan en menor medida las complicaciones y lo tomen y manejen como algo cotidiano.

Igualmente, con referencia a la segunda hipótesis de investigación se la da por aceptada, ya que se postuló que se encontraría la presencia de muchos estilos de afrontamiento aplicados. Con referencia a los estilos de afrontamiento al estrés más recurrentes, se encontró que el estilo de afrontamiento activo es el más usado por los choferes para buscar resolver directamente el problema entre ellos. Luego de él otro más usado es el de acudir a la religión, por ello es típico que los choferes tengan en sus carros mensajes alusivos a la religión o algún tipo de santo, mostrando su devoción por alguno de ellos. Si bien es cierto en la práctica puede que esto no sea visible, se ve reflejado principalmente en sus costumbres y creencias. Otro estilo de afrontamiento es el de razones instrumentales, es decir, buscar el apoyo directo de otras personas, de allí se comprende que entre los choferes se muestra mucha colaboración entre unos y otros cuando se pasa por algún tipo de altercado. Nótese, contrariamente, que los estilos menos usados hacen referencia al abordaje emocional y a la aceptación de la culpa. Es decir, la mayoría de los choferes toma en cuenta el locus de control externo, busca responsabilizar a los otros de los accidentes y poco a sí mismos. $\mathrm{Y}$ otro estilo poco usado es el de postergación del afrontamiento y evadir el afrontamiento. Complementariamente, un porcentaje promedio de conductores recurre a estrategias como desentendimiento mental, planificación, supresión de actividades competentes, negación. Estos estilos se caracterizan porque el chofer busca desentenderse de la situación, muchas veces influenciado por la imagen que el otro conductor les imparte, en la que ellos creen que pueden tomar ventajas, especialmente cuando con quien chocan son mujeres o conductores principiantes, donde los conductores experimentados consideran que pueden sacar partido de dicha situación.

También se encontró en los resultados que existe una correlación directa entre la ansiedad-rasgo y los estilos planificación de actividades, análisis de las emociones, negación. Esto quiere decir que a mayor ansiedad, existen mayores niveles de planificación de actividades, análisis de emociones y también negación de la problemática. Los niveles de ansiedad muchas veces hacen que las personas reaccionen de modo más productivo, al tener que recurrir al uso de todas sus potencialidades para poder resolver algunos problemas. En algunos casos, esto suele ser muy común entre los conductores cuando no quieren aceptar que no cometieron alguna imprudencia en algún choque o accidente, especialmente si la situación de tensión se ve en aumento por la presencia de algún efectivo policial. Por otro lado, se encontró una correlación inversa entre la ansiedadrasgo y los estilos búsqueda de soporte social, búsqueda de soporte emocional, reinterpretación positiva de la experiencia y distracción. Esto quiere decir 
que a mayores niveles de ansiedad, existen menores niveles de soporte social, soporte emocional, reinterpretación positiva de la experiencia y distracción. Aparentemente el perfil de los conductores no se caracteriza por el uso de apoyo social o emocional a la hora de la resolución de problemas. Salazar y Pereda (2010) tuvieron como objetivo conocer la relación entre el síndrome de Burnout y los patrones de comportamiento en contexto de tráfico, donde se concluyó que el síndrome de Burnout se relaciona significativamente con los patrones de comportamiento en contexto de tráfico en los conductores, justamente haciendo énfasis en cómo es que el estado de ánimo se ve más vulnerado en los contextos laborales, y por ende los estilos de afrontamiento se perciben más críticos.

\section{CONCLUSIONES}

Las conclusiones principales del estudio son las siguientes:

- Se encontró que a mayor ansiedad, existen mayores niveles de planificación de actividades, análisis de emociones, conductas inadecuadas y también negación de la problemática.

- Se encontró que a mayores niveles de ansiedad, existen menores niveles de soporte social, soporte emocional, supresión de actividades competitivas, reinterpretación positiva de la experiencia, aceptación y distracción.

- Finalmente, no se encontró correlación entre las estrategias de afrontamiento al estrés de afrontamiento directo, retracción del afrontamiento y retorno a la religión.

- Existen mayores porcentajes para las personas con un nivel de ansiedad medio, mientras que menos casos con un nivel de ansiedad alto.

- Los estilos que más predominaron en la muestra de estudio fueron: el estilo de afrontamiento "activo", seguido del estilo de afrontamiento de "acudir a la religión" y, ligeramente debajo, "búsqueda de apoyo social (razones instrumentales)". Los estilos menos usados fueron: "búsqueda de apoyo social (razones emocionales)" y "aceptación".

\section{NOTA DE RECONOCIMIENTO}

El autor reconoce la colaboración de los participantes en este estudio. 


\section{REFERENCIAS BIBLIOGRÁFICAS}

American Psychiatric Association (2003). DSM IV-TR, Breviario: criterios diagnósticos. Barcelona: Masson.

Carver, C. y cols. (1989). Cuestionario de Estrategias de Afrontamiento (COPE). Nueva York: Springer-Verlag.

Cassaretto, M (2009). Relación entre las cinco grandes dimensiones de la personalidad y el afrontamiento en estudiantes preuniversitarios de Lima Metropolitana. Tesis para optar por el grado académico de Magíster en Psicología con mención en Psicología Clínica y de la Salud, UNMSM. Lima, Perú.

Chávez, G. (2004). Estrategias de Afrontamiento a la Ansiedad de Evaluación y su Relación con el Desempeño Académico en Estudiantes Universitarios incorporados a Modelos Educativos Innovadores. Tesis para optar Maestría en Psicología Aplicada, Universidad de Colina. Colima, México.

Hernández, Fernández y Baptista (2006). Metodología de la investigación. México: Ediciones Mac. Graw-Hill. 10 Edi.

Lima, C. y Juárez, A. (2008). Un Estudio Exploratorio Sobre Estresores Laborales en Conductores de Transporte Público Colectivo en el Estado de Morelos, México. Revista Electrónica Ciencia \& Trabajo, pp. 126131. Obtenido el 15 de mayo del 2012, disponible en línea: http://www. cienciaytrabajo.cl/pdfs/30/pagina126.pdf

Ministerio de Transportes y Comunicaciones (2001). Reglamento Nacional de Tránsito. Aprobado mediante el Decreto Supremo N 033-2001-MTC. Lima, Perú.

OMS (2004). Informe Mundial sobre Prevención de los Traumatismos Causados por el Tránsito: Resumen. Obtenido el 15 de mayo del 2012, disponible en línea: http://www.paho.org/spanish/dd/pub/resumen_informe_mundial_ traumatismos.pdf

Pardo, F. (2010). Bienestar Psicológico y Ansiedad Rasgo-Estado en alumnos de una MBA de Lima Metropolitana. Tesis para optar el título de Licenciado en Psicología con mención en Psicología Clínica, PUCP. Lima, Perú.

Ponce, C., Bulnes, M., Aliaga, J., Delgado, E. y Solís, R. (2006). Estudio psicológico sobre los patrones de conducta en contextos de tráfico, en grupos de automovilistas particulares y profesionales de Lima Metropolitana. Revista de Investigación en Psicología, Universidad Nacional Mayor de San Marcos, pp. 33-64. 